\title{
A novel through-the-scope twin clip traction-assisted endoscopic submucosal dissection in an in vitro pig stomach model
}

A novel through-the-scope twin clip (TTS-TC) was developed for the closure of large gastrointestinal tract wounds $[1,2]$. In the current study, we initially explored the application of TTS-TC traction-assisted endoscopic submucosal dissection (ESD) in an in vitro pig stomach model.

TTS-TC traction-assisted gastric ESD was performed as follows ( Video 1 ). (i) First, the mucosa was marked at the greater curvature of the stomach, and submucosal injection was performed, followed by mucosal incision along the marked points ( $\mathbf{F i g} \mathbf{1}$ a). (ii) A TTS-TC was delivered through the endoscopic working channel (3.2-mm inner diame-

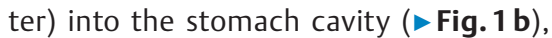
and the clip on one side of the TTS-TC was opened by controlling the handle to clamp the oral side of the incised mucosa

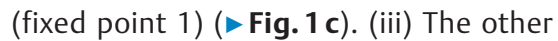
side of the TTS-TC was opened to clamp the mucosa of the anterior wall of the lesser curvature of the stomach on the opposite side of the incised mucosa (fixed point 2) (> Fig.1d). (iv) The submucosa was fully exposed through the mutual traction between the two fixed points ( Fig.1e), and an endoscopic knife was then used to dissect the submucosa.
Only one TTS-TC was used to complete the mucosal traction. The size of the dissected mucosa was $3.8 \times 2.5 \mathrm{~cm}$. The total operation time was 19.6 minutes, with the time taken to dissect the submucosa being 8 minutes. The TTS-TC did not detach from the two fixed sites during dissection of the submucosa. The wound was completely closed with one TTS-TC and three conventional throughthe-scope clips (TTSCS), with the time taken for wound closure being 4 minutes. The TTS-TC is a promising device for mucosal traction to assist gastric ESD.

Endoscopy_UCTN_Code_TTT_1AO_2AG
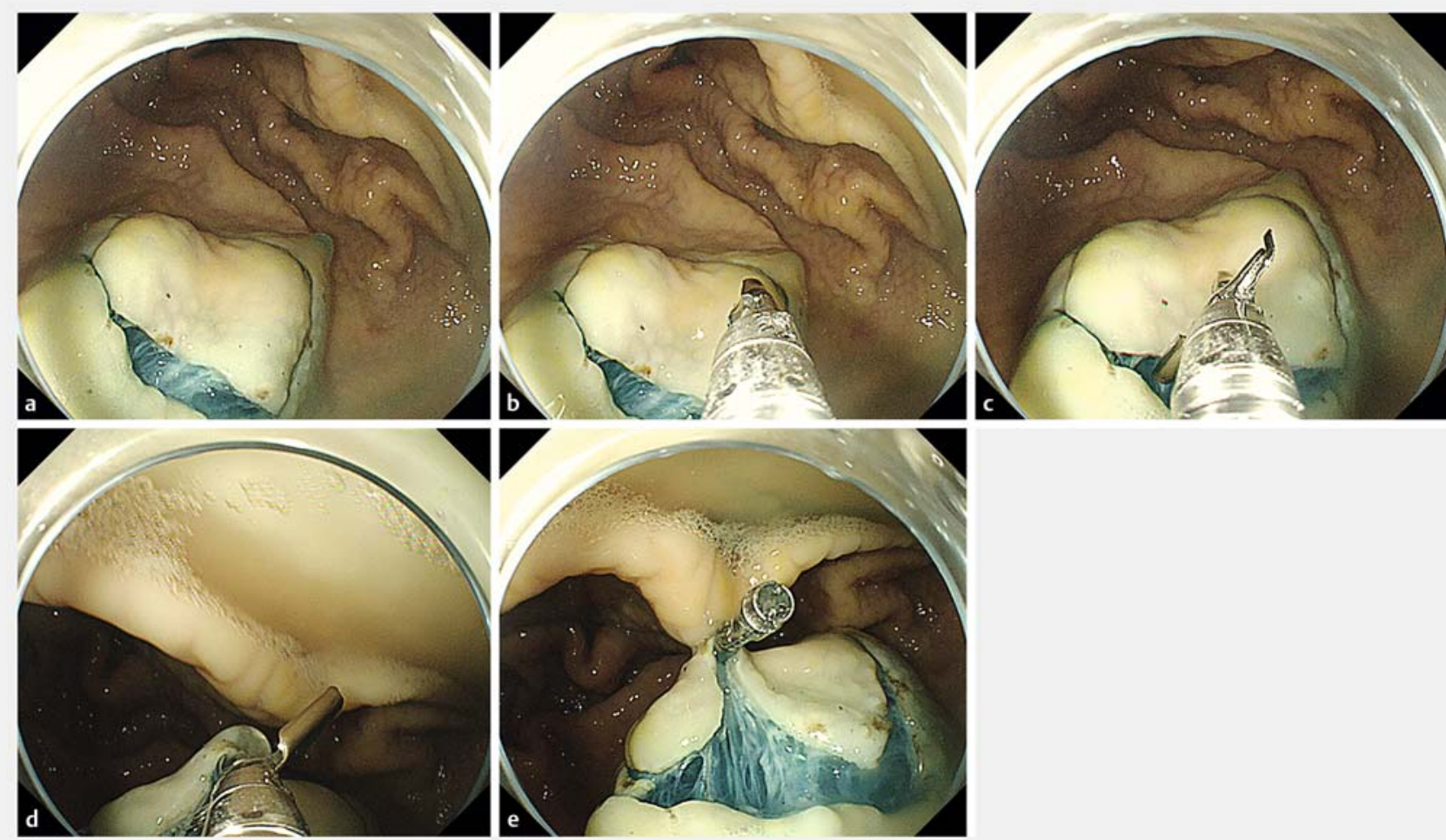

- Fig. 1 Endoscopic views showing: a the incised mucosa at the greater curvature of the stomach; $\mathbf{b}$ a through-the-scope twin clip (TTS-TC) being delivered into the stomach cavity through the endoscope working channel; $\mathbf{c}$ the oral side of the incised mucosa being clamped by one side of the TTS-TC (creating fixed point 1); $\mathbf{d}$ the clamped mucosa being pulled close to the mucosa of the anterior wall of the lesser curvature of the stomach, and the mucosa of the anterior wall being clamped by the other side of the TTS-TC (creating fixed point 2); e the submucosa being fully exposed using the mutual traction between the two fixed points. 


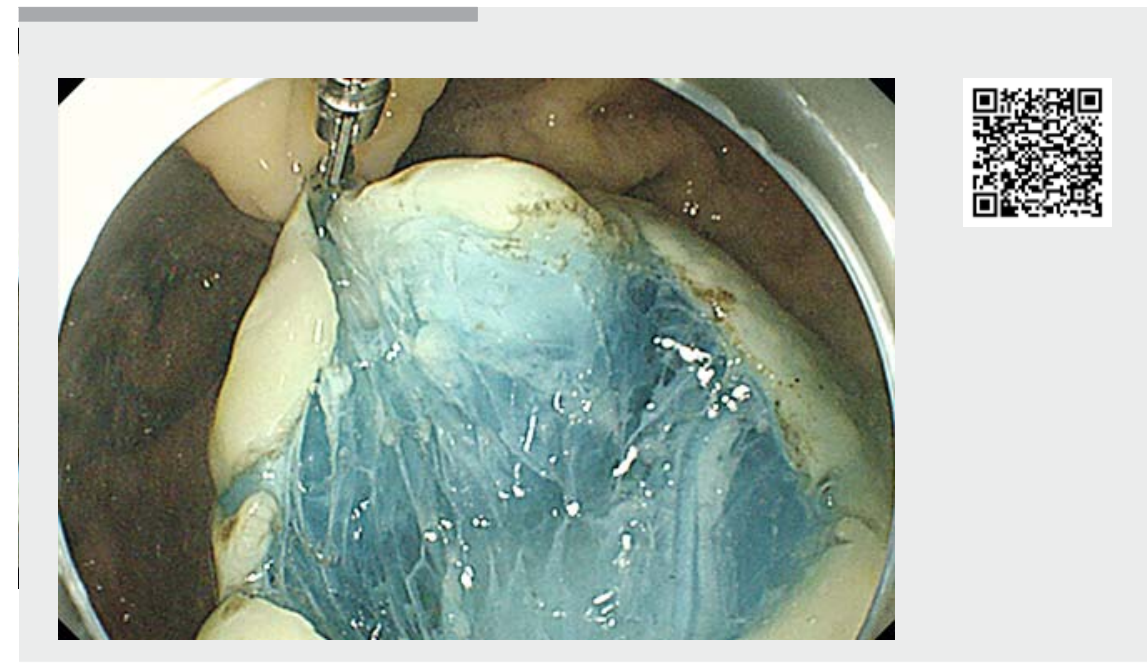

Video 1 A example of through-the-scope twin clip (TTS-TC) traction-assisted endoscopic submucosal dissection in an in vitro pig stomach model, with subsequent closure of the wound using the TTS-TC.

\section{Competing interests}

The authors declare that they have no conflict of interest.

The author

\section{Qiang Zhang}

Guangdong Provincial Key Laboratory of Gastroenterology, Department of Gastroenterology, Nanfang Hospital, Southern Medical University, Guangzhou, Guangdong Province, China

\section{References}

[1] Zhang Q, Wang Z, Bai Y. A novel throughthe-scope twin endoclip for a large mucosal closure in a live pig model. Endoscopy 2019; 51: E372-E373

[2] Qiang Zhang, Zhen Wang. Endoscopic closure of a large-size perforation using a novel through-the-scope twin endoclip in an ex vivo porcine stomach model. Dig Endosc 2020; 32: e61-e62

Bibliography

Endoscopy 2021; 53: E259-E260

DOI 10.1055/a-1247-4619

ISSN 0013-726X

published online 23.9.2020

(c) 2020. Thieme. All rights reserved.

Georg Thieme Verlag KG, Rüdigerstraße 14,

70469 Stuttgart, Germany

\section{ENDOSCOPY E-VIDEOS}

https://eref.thieme.de/e-videos

\section{Qiang Zhang, MD}

Guangdong Provincial Key Laboratory of Gastroenterology, Department of Gastroenterology, Nanfang Hospital, Southern Medical University, Guangzhou, Guangdong Province 510515, China 4024313@qq.com 